\title{
Dynamic Object and Target based Gaming CAPTCHA for Better Security Analysis
}

\author{
Vipin Kumar \\ Oriental Institute of Science \& \\ Technology, Bhopal
}

\author{
Atul Barve \\ Oriental Institute of Science \& \\ Technology, Bhopal
}

\begin{abstract}
A technique which is used to identify whether user is human or robot is called CAPTCHA. This technique has been using from last few decades for security concern, it is a kind of Turing test. Usually, distorted alphabets are generally used for this kind of test, because it has been known that, it is easy to analyze the distorted letters by human but not by robot or bots. But now there are so many intelligent machines which can recognize these alphabets, so the security has been broken. Then there are so many researchers have been made over this security, like OTP based, moving letters, 3d letters and gaming CAPTCHA. But somewhere somehow they are lacking with their complexities or breakable approach. Gaming CAPTCHA was the best and modern approach for providing better security in the field of CAPTCHA. But the gaming level is weak with static targets or objects, which may be breakable for somehow because robot will have infinite time to break game by analyzing and targeting static objects and targets. So to overcome this problem a new promising concept has been proposed in this paper i.e. "Dynamic Object \& Target based Gaming CAPTCHA for Better Security Analysis", it is most attractive and cognitive way in the world of CAPTCHA. This CAPTCHA will have dynamic target and objects, it means that target and object will be in motions and user will be asked to drag and drop these moving object to the moving target even within limited time or session, which is easily possible for human but almost impossible for robot.
\end{abstract}

\section{Keywords}

CAPTCHA, Drag and Drop, Game, Session, Time, etc.

\section{INTRODUCTION}

There are so many CAPTCHA techniques exist, some of them are very easy for human as well as for robot and some of them are very hard for robot to solve as well as for human. So we require a technique which is very easier for human and almost impossible for robot. This paper is based on advanced gaming CAPTCHA and this CAPTCHA game is differ from the existing one because all the object and target will be in motion and you will be asked to drag and drop these moving object. It is very easy for human but almost impossible for robot. And you will also get time limit, it means that you will have 15 seconds to solve it and if you are human then you can solve it within 8 seconds. In this system your CAPTCHA would be expired if you do not submit your form till 2 minutes and also page expiration when your page is not active for 3 minutes, your page session will be restarted and you will have to perform CAPTCHA verification again then you can submit your form. This is the new promising concept in the field of CAPTCHA.

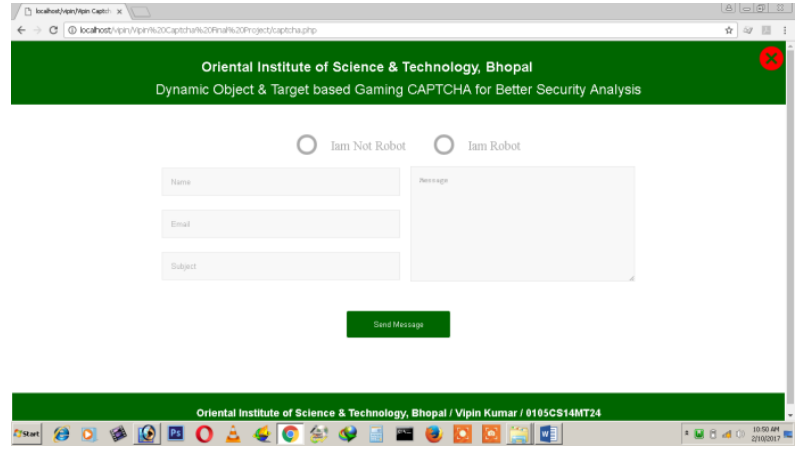

Fig. 1.1 Proposed Gaming CAPTCHA

\section{BASE PAPER}

Gaming the game: Defeating a game CAPTCHA with efficient and robust hybrid attacks proposed by Song Gao, Manar Mohamed, NiteshSaxena and Chengcui Zhang in IEEE 2014. [7]

This is an advance CAPTCHA based on gaming. You will get an artificial problem to solve like a game. You will be asked to drag and drop the desired object to the target place where you asked to put. This is advanced and most promising technique. But the problem is that a robot will have sufficient time to break this CAPTCHA, and the target as well as object are static in position. These static object can be cracked by modern intelligent systems.

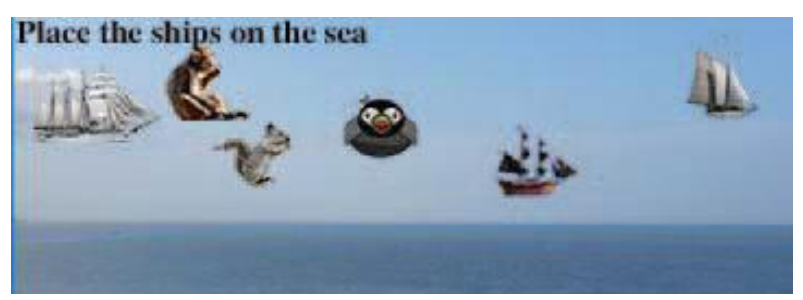

Fig. 2.1 Present Gaming CAPTCHA

In the above CAPTCHA, you are asked to put ship on the sea by drag and drop method.

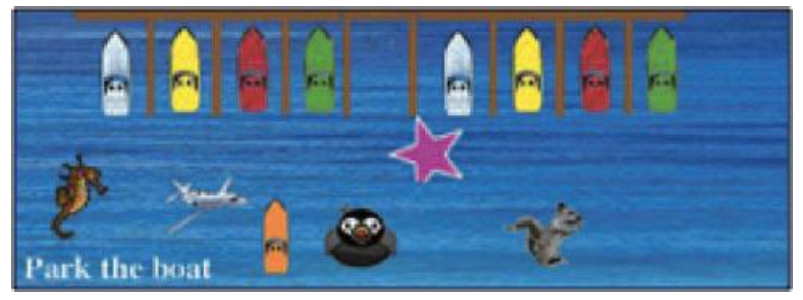

Fig. 2.2 Present Gaming CAPTCHA

In the above CAPTCHA, you are asked to park ship on right place by drag and drop method. 


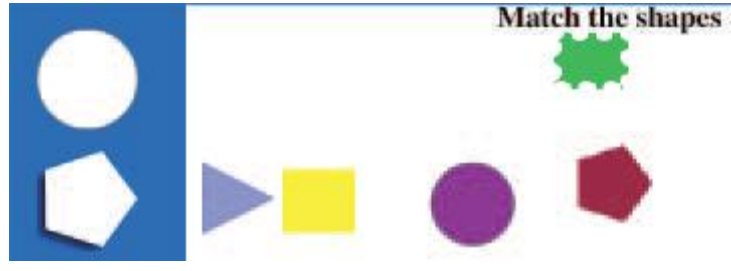

Fig. 2.3 Present Gaming CAPTCHA

In the above CAPTCHA, you are asked to match the desired shave with the given one by drag and drop method.

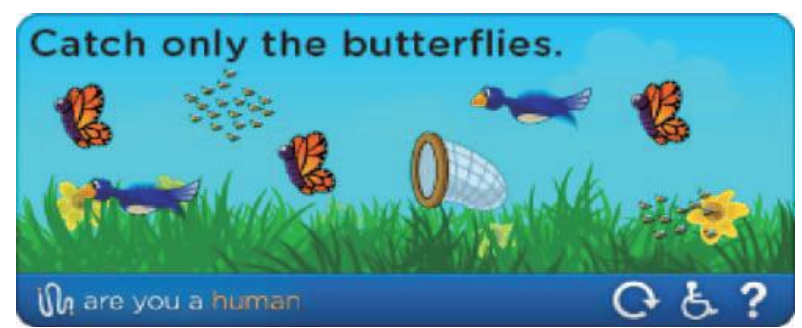

Fig. 2.4 Present Gaming CAPTCHA

In the above CAPTCHA, you are asked to catch butterflies only by drag and drop method.

This CAPTCHA technique has higher potential of security but it is also breakable by intelligent machines because object and target position are static and a robot will have infinite time to solve it. This is the drawback of this system. And there are so many systems exist for CAPTCHA verification but they are lacking either to understand by human or breakable for robot which is required to overcome.

\section{PROPOSED WORK}

Here it is the solution which proposes a dynamic object \& target gaming based CAPTCHA, it means that here the target is not static as well as object, you will have to drag \& drop moving object to moving target which is considered impossible for robot in given time, because we are also using a CAPTCHA session, you will get 10-15 seconds to achieve your goal and it can be achieved by 5 to 8 seconds by human or may be less. But not possible by robot because after given session time another CAPTCHA will be appeared to achieve. So this technique is based on totally dynamic features and sessions which is very easy for human but almost impossible by robots. This is the newest way in the world of gaming CAPTCHA.

Let's have an example for the proposed system. Here it is the real world background of forest where few animals like elephant, giraffe and bird are present and there is a well in the forest. These animals are walking towards well and cross it within 15 seconds. You will be asked to drag and drop one the animal like elephant, as the figure given below you have been asked to drag and drop elephant in the well before crossing the well or within 15 seconds because if you are human then it can be solved within 5-8 seconds, but if you are robot then this 15 seconds is not sufficient to crack it. But breaking this CAPTCHA is not quite easy because it is not a static game where target and object's position is static and you will have time limit to break it, here object as well as target are moving, there is no static positions, every second the position is getting changed. It is understandable for human very easily but impossible for robot especially in 15 seconds. After 15 seconds you will get another CAPTCHA to solve but not like the previous one, it will change the target as well as object.

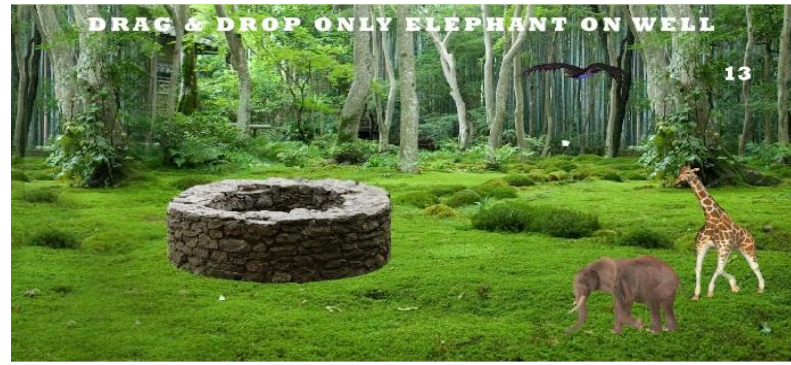

Fig. 3.1 Advanced Gaming CAPTCHA

First of all you will get a page where you will be asked to perform CAPTCHA verification then fill your form and submit it. But if you try to submit form before verification then it will show you a message i.e. CAPTCHA verification not done.

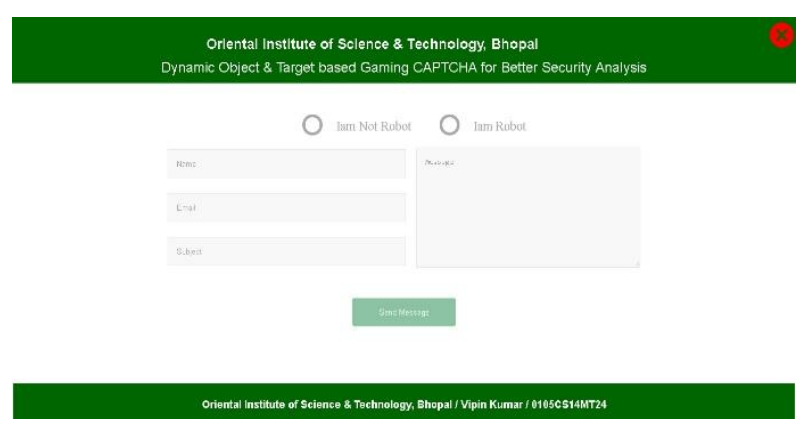

Fig. 3.2 CAPTCHA Verification Page

In the above figure i.e. Fig 3.2, there are two radio buttons i.e. I am not robot and I am robot, when you click on I am robot then a message will come i.e. Invalid attempt, it is only to show you the difference. But when you click on I am not robot then a CAPTCHA verification game will be appeared.

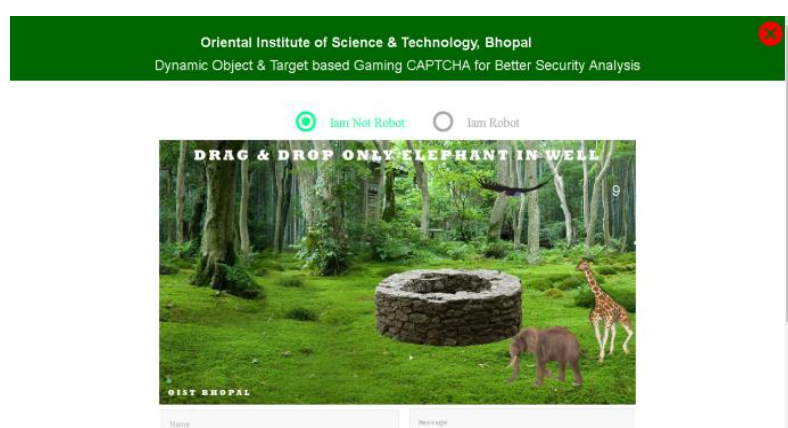

Fig. 3.3 CAPTCHA Game

If you do not perform CAPTCHA verification and try to submit empty form then it will not allow you to submit empty form.

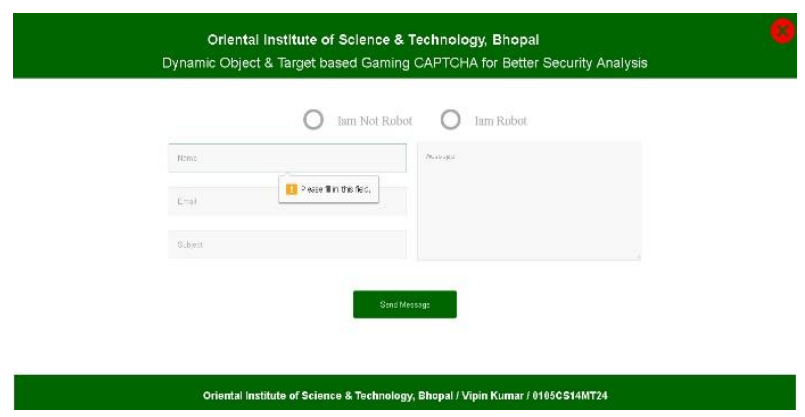

Fig. 3.4 Field Validation 
And if you filled form and try to submit without CAPTCHA verification then a message will appear i.e. CAPTCHA verification not done.

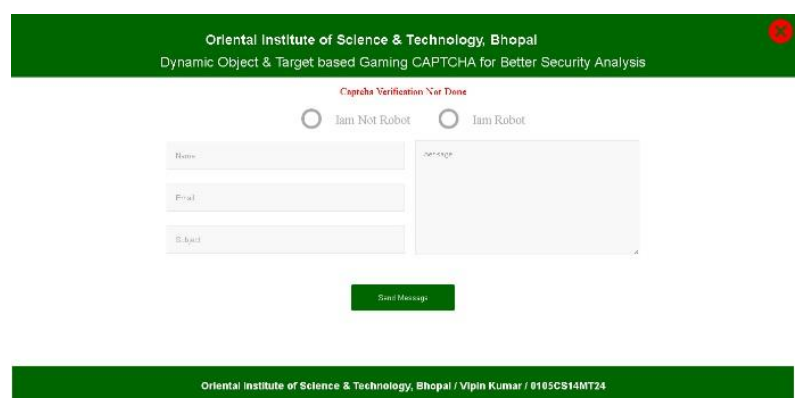

\section{Fig. 3.5 CAPTCHA Validation}

When you click on 'I am not a robot' then a game will appear where you will have to drag and drop the desired object.

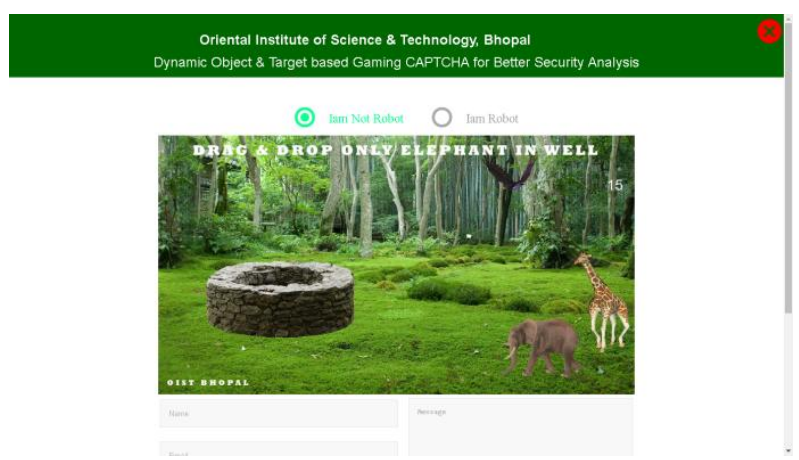

Fig. 3.6 CAPTCHA Game Active

If you do not solve CAPTCHA within 15 seconds then it will be expired and you will have to reload CAPTCHA again.

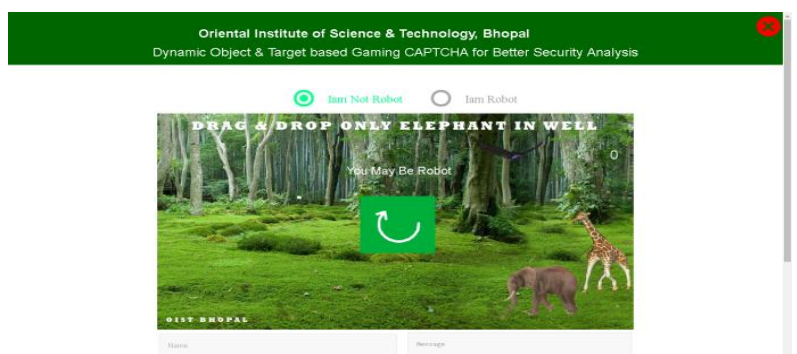

Fig. 3.7 CAPTCHA Reload

When you click on reload button then a new CAPTCHA will appear to solve.

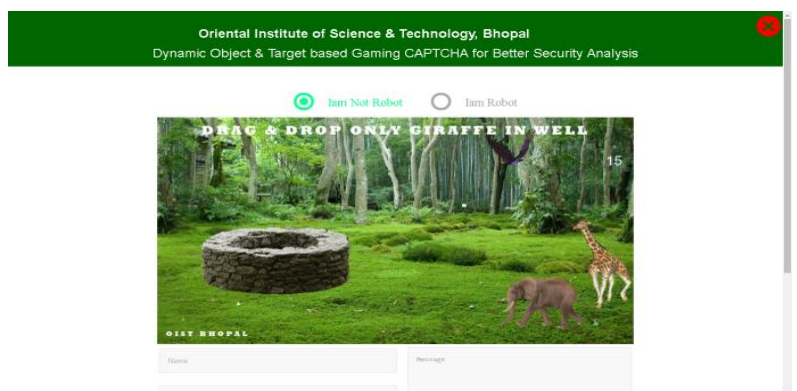

Fig. 3.8 Reloaded New CAPTCHA

But when you solved it within 15 seconds then you will get a message on screen i.e. CAPTCHA verification successful.

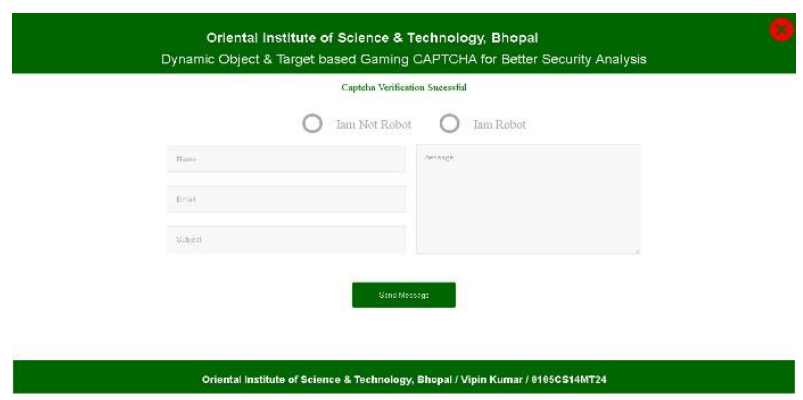

Fig. 3.9 CAPTCHA Verification Successful

After verification you can submit form and after submitting form you will get a success message.

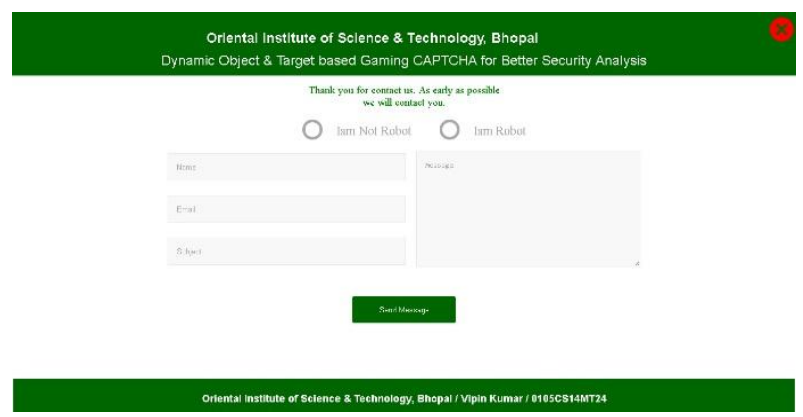

Fig. 3.10 Form Submitted Successfully

After submitting a form successfully if you try to submit form again without performing CAPTCHA again then you will not be allowed to submit form, you will get a message i.e. CAPTCHA verification not done.

When you solved CAPTCHA successfully and not submitting form till 2 minutes then your CAPTCHA will be expired.

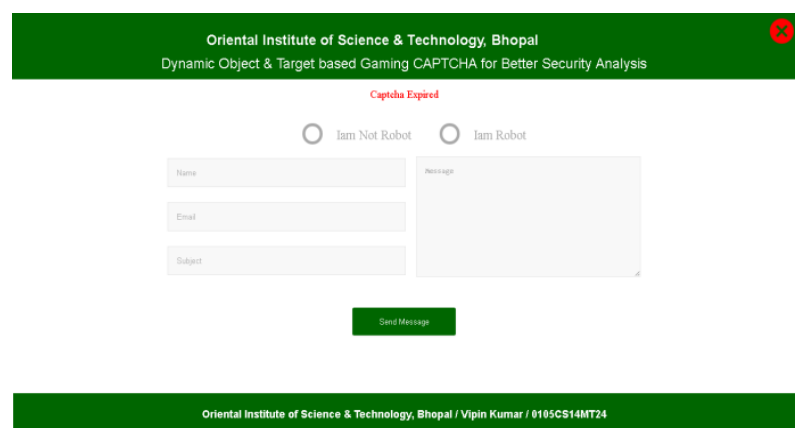

Fig. 3.11 CAPTCHA Expired

And if there is activity till 3 minutes then the page session will restarted.

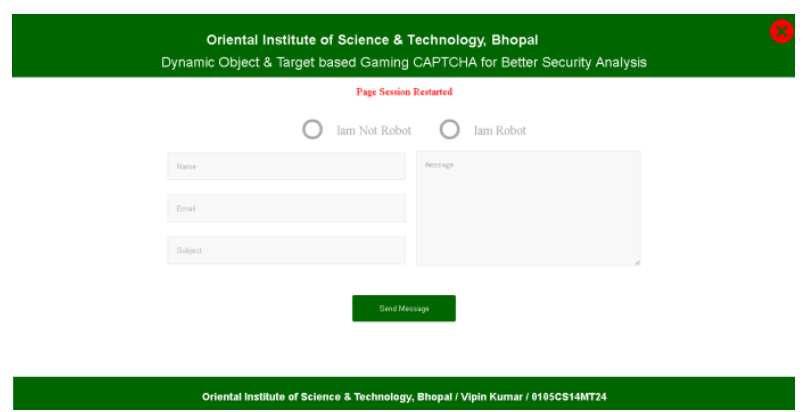

Fig. 3.12 Page Session Expired

When you click on I am robot then you will get a message 'Invalid Attempt'. 


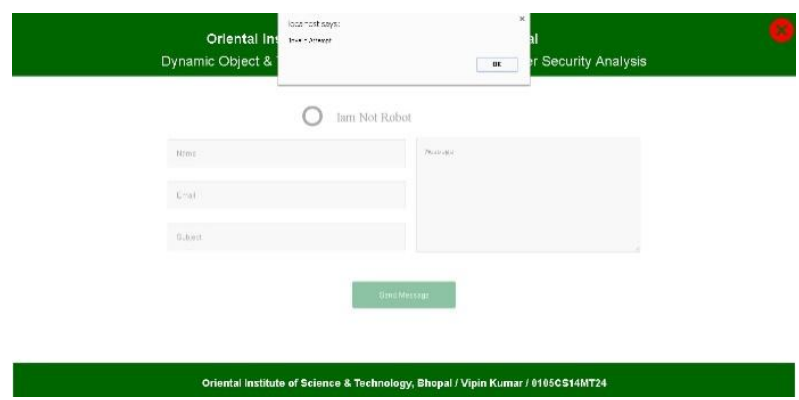

Fig. 3.13 Invalid Attempt

When you click on close button which is situated in the upper right corner of the webpage then session token will be expired and if you want to perform CAPTCHA verification again or want to submit form again then you will have to start session again.

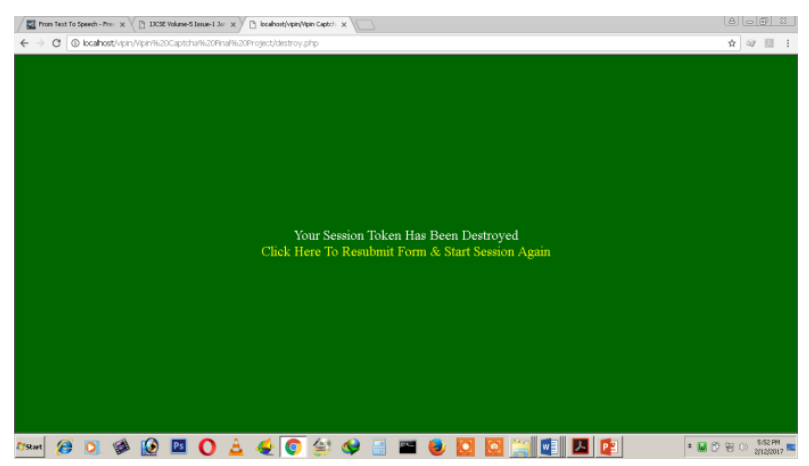

Fig. 3.14 Session Token Expired

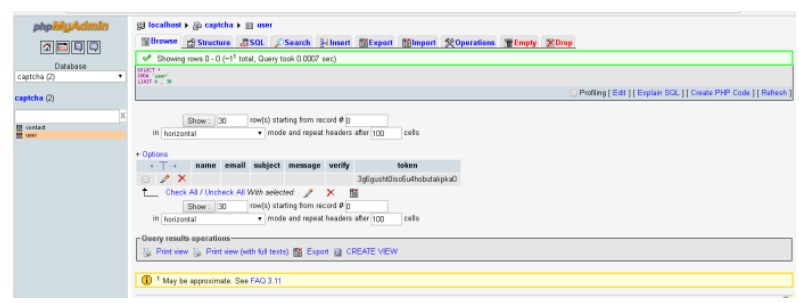

Fig. 3.15 MySQL Database

The CAPTCHA is designed in action script and no one can extract it from webpage. The flow chart is given below which express flow details.

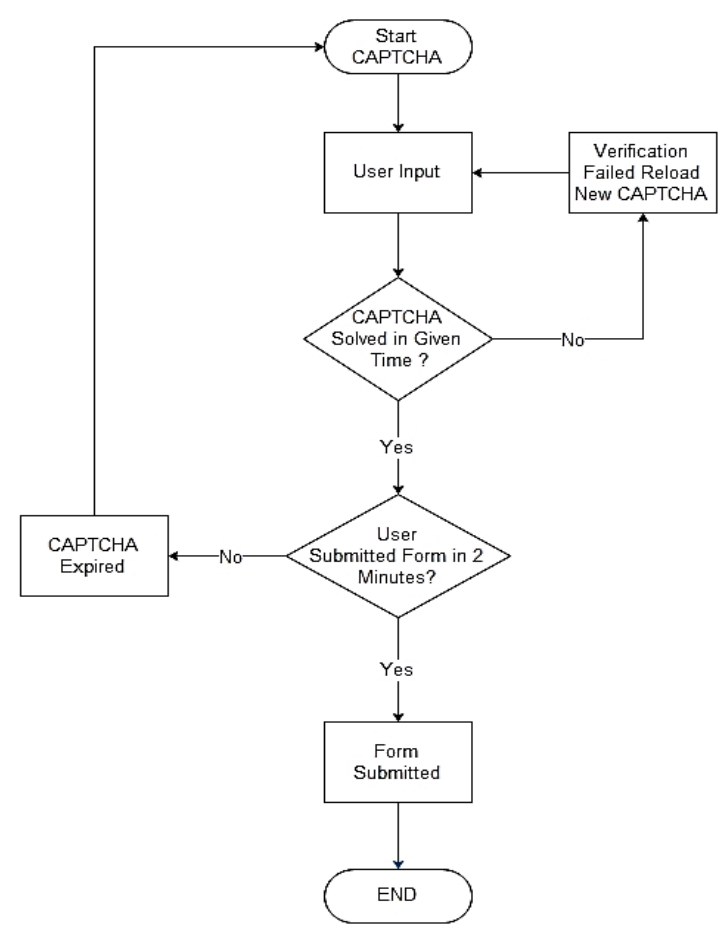

Fig 3.16: Flow Chart

4. RESULT ANALYSIS

\begin{tabular}{l|c|c|} 
& $\begin{array}{c}\text { Existing } \\
\text { method }\end{array}$ & $\begin{array}{c}\text { Proposed } \\
\text { method }\end{array}$ \\
\hline $\begin{array}{l}\text { Time to } \\
\text { Solve } \\
\text { CAPTCHA }\end{array}$ & $\begin{array}{c}\text { No Tims } \\
\text { Limit }\end{array}$ & $\begin{array}{c}15 \text { Seconds } \\
\text { per } \\
\text { CAPTCHA }\end{array}$ \\
$\begin{array}{l}\text { T arget \& } \\
\text { Object } \\
\text { Position }\end{array}$ & Static & Dynanic \\
\hline $\begin{array}{l}\text { Completion } \\
\text { T ime }\end{array}$ & $5-8$ Seconds & $5-8$ Seconds \\
\hline $\begin{array}{l}\text { CAPTCHA } \\
\text { Session } \\
\text { Page } \\
\text { Session }\end{array}$ & No & Yes \\
\hline $\begin{array}{l}\text { Game's } \\
\text { Graphics }\end{array}$ & No & Yes \\
& &
\end{tabular}

Fig. 4.1 Comparison Chart

When I implemented the proposed work on the system it has 15 minutes to solve the CAPTCHA, but a legitimate human can solve CAPTCHA in 5-8 seconds easily.

\section{CONCLUSION}

Thus the proposed system is able to provide best security in the field of CAPTCHA. It is non-breakable gaming CAPTCHA which has session, dynamic targets, dynamic objects and many more. This is the advance level of gaming CAPTCHA with more artificial interactions which cannot be cracked by robots. Hence it is the best way in the world of gaming CAPTCHA. 


\section{FUTURE SCOPE}

The current proposed concept of Dynamic Object \& Target based Gaming CAPTCHA for Better Security Analysis definitely get enhanced in future because we can also develop more interactive and intelligent game through which we can make system stronger.

\section{REFERENCES}

[1] Vipin Kumar, Atul Barve, "A survey on existing CAPTCHA techniques \& proposed gaming CAPTCHA for better security analysis" IJCSE Vol-5, Issue 1, 2017

[2] Jing Song Cui, Li Jing Wang, JingTing Mei, Da Zhang, Xia Wang, Yang Peng, WuZhou Zhang, "CAPTCHA Design Based on Moving Object Recognition Problem" IEEE 2009.

[3] Jing-Song Cui, Jing-Ting Mei, Xia Wang, Da Zhang, Wu-Zhou Zhang "A CAPTCHA Implementation Based on 3D Animation", International Conference on Multimedia Information Networking and Security of IEEE, Nov 2009

[4] Ibrahim FurkanInce, YucelBatu Salman, Mustafa Eren Yildirim and Tae-Cheon Yang,"EXECUTION TIME PREDICTION FOR 3D INTERACTIVE CAPTCHA BY KEYSTROKE LEVEL MODEL", Fourth International Conference on Computer Sciences and Convergence Information Technology of IEEE, 2009.

[5] Chun-Ming Leung, "Depress Phishing by CAPTCHA with OTP", international conference of IEEE, 2009.

[6] Jing-Song Cui, Jing-Ting Mei, Xia Wang, Da Zhang, Wu-Zhou Zhang in "A CAPTCHA Implementation Based on 3D Animation", international conference of IEEE,2009

[7] Wai Chow1, Willy Susilo2, Hua-Yu Zhou1, "CAPTCHA Challenges for Massively Multiplayer Online Games", International Conference on Cyber worlds of IEEE,2010
[8] Jing-Song Cui, Jing-Ting Mei, Wu-Zhou Zhang, Xia Wang, Da Zhang, "A CAPTCHA Implementation Based on Moving Objects Recognition Problem", international conference of IEEE,2010

[9] Ryan C. McDaniel and Roman V. Yampolskiy, "Embedded Non-Interactive CAPTCHA for Fischer Random Chess", international conference of IEEE,2011

[10] Aadhirai R, Sathish Kumar P J and Vishnupriya S, "Image CAPTCHA: Based on Human Understanding of Real World Distances" ,IEEE Proceedings of 4th International Conference on Intelligent Human Computer Interaction, Kharagpur, India, December 27-29, 2012.

[11] Rahul Saha 1, Riyanka Manna3 and Dr. G. Geetha 2 "CAPTCHINO- A Gamification of Image-based CAPTCHAs to Evaluate Usability Issues ", international conference of IEEE,2012

[12] Song Gao, Manar Mohamed, Nitesh Saxena, Chengcui Zhang, "Gaming the game: Defeating a game CAPTCHA with efficient and robust hybrid attacks", IEEE 2014

[13] Manar Mohamed, Song Gao, Nitesh Saxena, and Chengcui Zhang, "Dynamic Cognitive Game CAPTCHA Usability and Detection of Streaming-Based Farming", 2014.

[14] Seyed Mohammad Reza SaadatBeheshti, PanosLiatsis, "How Humans Can Help Computers to Solve an Artificial Problem", international conference of IEEE 2015.

[15] ArtemShumilov, Andrey Philippovich, "Cloud-Based CAPTCHA Service”, IEEE, 2016.

[16] Luis von Ahn, Manuel Blum, Nicholas J Hopper, John Lanford "CAPTCHA : Using Hard AI Problems for Security", Carnegie Mellon University 